\title{
Transverse sinus mass misinterpreted as the source of cardiac emboli
}

\author{
Hassan H. Allam ${ }^{1}$, Abdulhalim J. Kinsara ${ }^{2}$, Amtalkhaliq Alrajawi', Tareq Tuaima ${ }^{1}$, Olga Vriz ${ }^{3}$ \\ ${ }^{1}$ King Abdullah Medical Complex, Ministry of Health, Jeddah; ${ }^{2}$ Department of Cardiology, Ministry of National \\ Guard-Health Affairs, King Saud Bin Abdulaziz University for Health Sciences, COM-WR, King Abdullah \\ International Medical Research Center, Jeddah; ${ }^{3}$ Heart Centre, King Faisal Specialist Hospital and Research Center, \\ Alfaisal University, Riyadh, Saudi Arabia
}

\begin{abstract}
Due to the proximity of the transvers sinus (TS) to the left atrial appendage (LAA) and pulmonary veins (PV), a mass in the TS can be misinterpreted as a LAA or PV thrombus, and considered as a
\end{abstract}

Correspondence: Abdulhalim Jamal Kinsara, Department of Cardiology, Ministry of National Guard-Health Affairs, King Saud Bin Abdulaziz University for Health Sciences, COM-WR, King Abdullah International Medical Research Center, Jeddah, Saudi Arabia. Tel. +966.569.968182. E-mail: akinsara@yahoo.com

Conflict of interest: The authors declare that they have no competing interests, and all authors confirm accuracy.

Funding: The study did not receive specific funding, but was performed as part of the employment of the authors, at King Saud bin Abdulaziz University for Health Sciences.

Key words: Transverse sinus mass; cardiac emboli; left atrial appendage mass.

Contributions: HHA, AR, TT, AJK, OV, conceptualization, methodology, data curation, manuscript original draft; HHA, AR, TT, formal analysis; AJK, supervision, project administration. All the authors have read and approved the final version of the manuscript and agreed to be accountable for all aspects of the work.

Declaration of patient consent: The authors certify that they have obtained patient consent forms. The patient's name and initial would not be published and conceal his identity.

Availability of data and materials: The data used to support the findings of this study are restricted by the King Abdullah International Medical Research Center Institution Review Board in order to protect patient's privacy. Data are available from the Institutional Review Board irb@ngha.med.sa or via the corresponding author, for researchers who meet the criteria for access to confidential data.

Received for publication: 4 September 2020.

Accepted for publication: 13 November 2020.

${ }^{\circ}$ Copyright: the Author(s), 2021

Licensee PAGEPress, Italy

Monaldi Archives for Chest Disease 2021; 91:1597

doi: 10.4081/monaldi.2021.1597

This article is distributed under the terms of the Creative Commons Attribution Noncommercial License (by-nc 4.0) which permits any noncommercial use, distribution, and reproduction in any medium, provided the original author(s) and source are credited. source of emboli in a patient with stroke or transient ischemic attack. The incorrect identification of a mass as a LAA thrombus would initiate unnecessary anticoagulation therapy or potentially, an evaluation for the excision of the mass if there is a concern about dislodgement. We are presenting a case illustrating this confusion and review the literature for similar cases.

\section{Introduction}

The transverse sinus (TS) is open at both ends and formed by the reflection of the visceral serosal pericardium from the posterior aspects of the aortic and pulmonary trunks over to the anterior aspect of the atrium [1]. Thus, a finger in the transverse sinus will pass behind the aortic and pulmonary trunks but in front of the superior vena cava on the right and the left atrial appendage (LAA) on the left [2]. A Transesophageal Echocardiography (TEE) in the mid esophageal view can, in most cases, distinguish the location of a TS mass, without the need for further investigation (CT or MRI).

\section{Case Report}

A 50-year-old diabetic woman presented with left sided numbness. The patient was admitted in Neurology for further investigation related to stroke of TIA. The clinical examination revealed that the patient was hemodynamically stable, with no motor weakness. The cardiac examination revealed a wide fixed second heart sound, and an ejection systolic murmur on the upper left sternum $2 / 6$. The carotid was normal. The ECG was in sinus rhythm with Right Bundle Bransh Block. The laboratory report indicated a normal CBC and renal function. The HgbAlc was 7.5 with the LDL $4 \mathrm{mmol} / \mathrm{L}$.

The TTE indicated a normal LV systolic function, normal valve, and LAVI $43 \mathrm{ml} / \mathrm{m}^{2}$. The right side of heart was dilated with rise in the pulmonary artery pressure, with Resting PASP, $45 \mathrm{~mm} / \mathrm{hg}$. The TEE was done as a workup for stroke and revealed a mobile mass in the transverse sinus (TS) (Figure 1). The differential diagnosis was a LAA thrombus or PV mass versus fat in the TS (Figure 2). However, the different mid-esophageal views assisted in the distinction of the exact location of the mass, avoiding the initiation of unnecessary interventions.

\section{Discussion}

The incorrect identification of a mass as a LAA thrombus, would cause the initiation of unnecessary anticoagulation thera- 
py or potentially, an evaluation for the excision of the mass if there is a concern about dislodgement. A TEE can reliably distinguish the location of a TS mass, avoiding the initiation of unnecessary investigations or interventions [3]. To identify a mass (fat) in the transverse sinus, rotating the transducer will document that it is within the transverse sinus and not the LAA.
Transverse sinus fat does not need any intervention for itself, but it is associated with an increased severity of coronary artery disease which necessitates more risk factor control, especially in the context of our patient being a diabetic and dyslipidemic [4]. There is similar case of a transverse sinus mass mistaken as a possible aortic abscess [5].

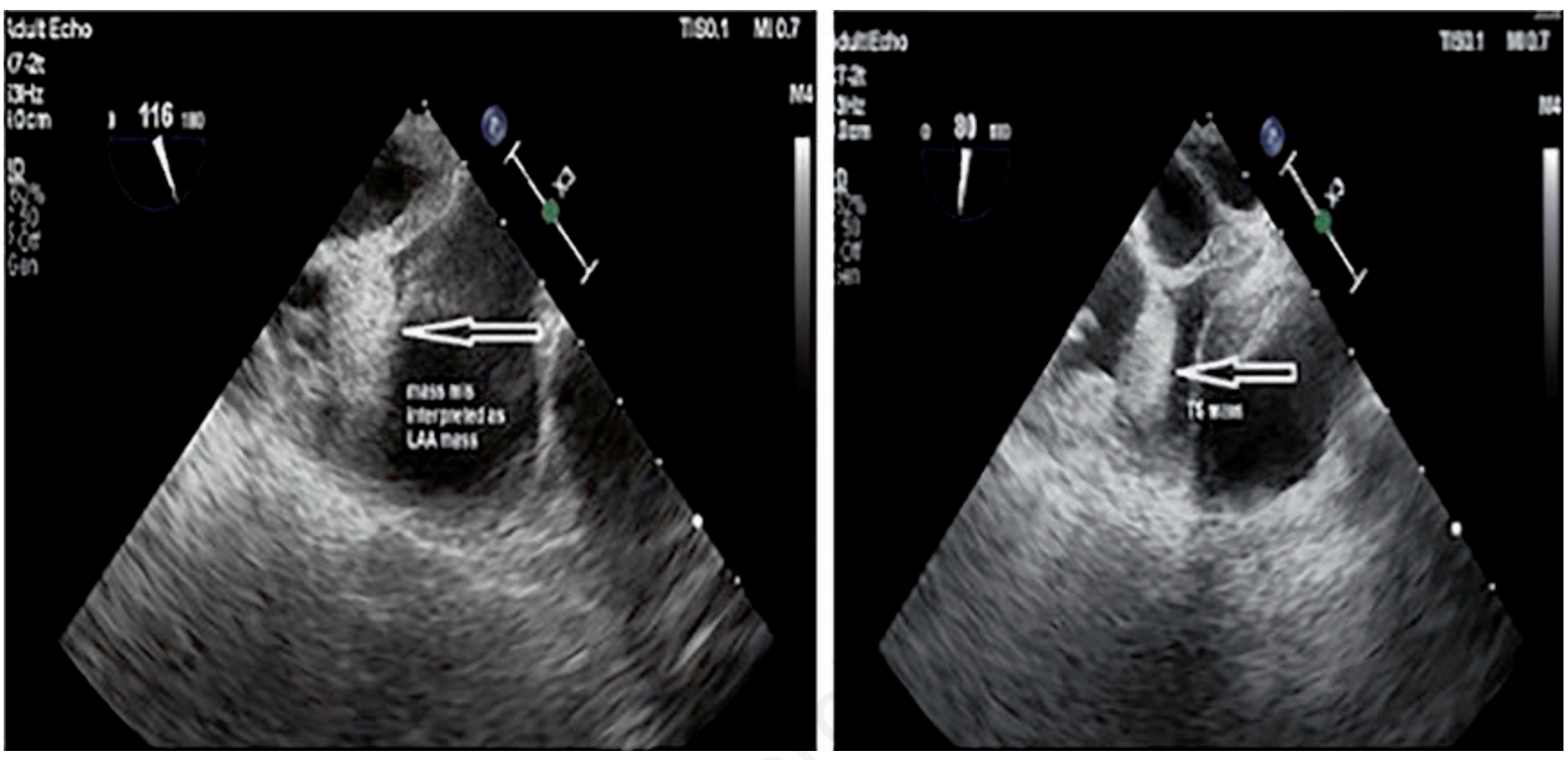

Figure 1. Mass in transverse sinus, thought to be a left atrial appendage thrombus.

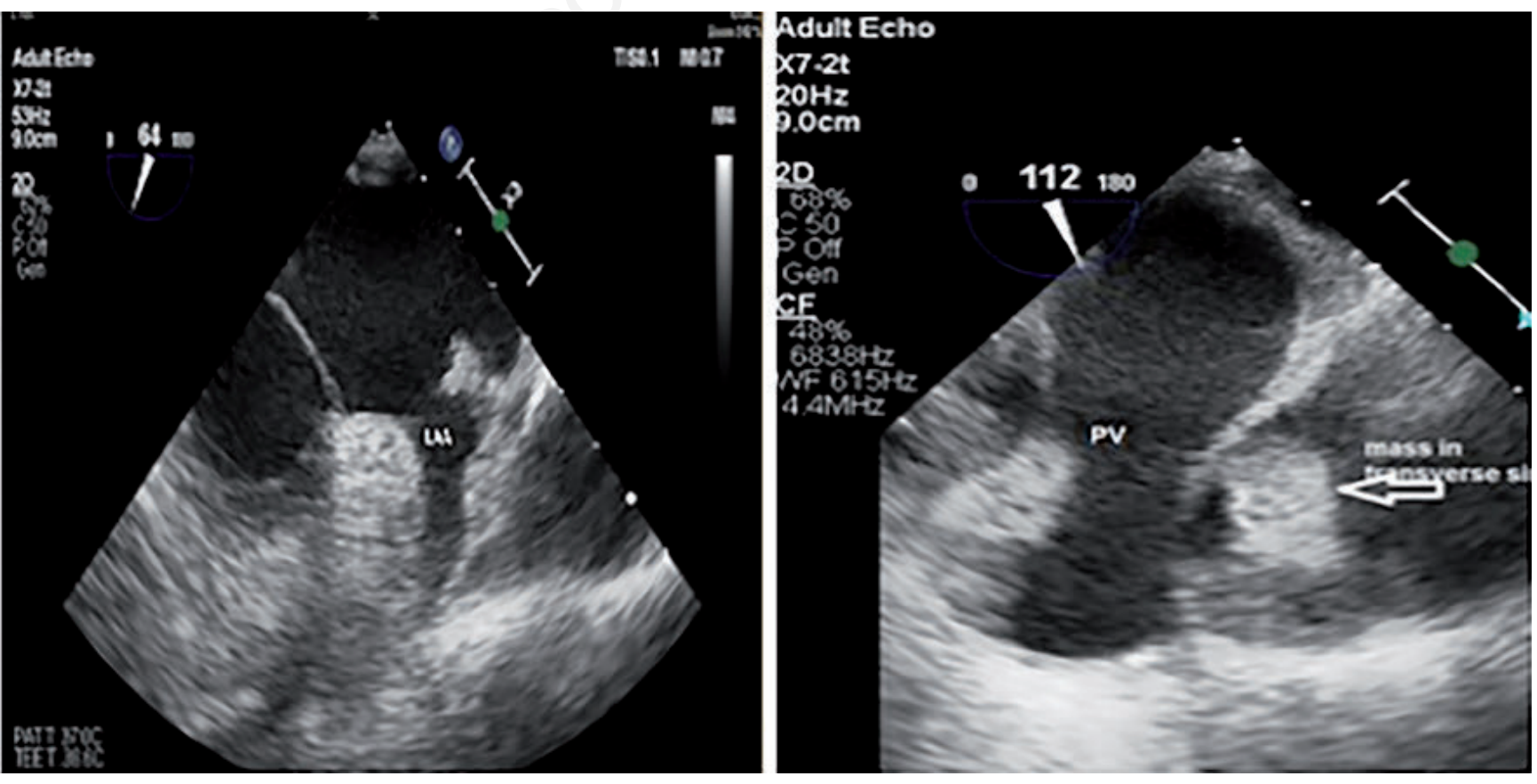

Figure 2. A focus left atrial appendage view showing clean LAA and pulmonary vein. 


\section{Conclusions}

Transvers sinus fat might mimics a left atrium thrombus and a special attention to the probe direction of transesophageal echocardiography will differentiate between transvers sinus fat and a left atrial thrombus or mass and avoid unnecessary anticoagulation or further diagnostic imaging

\section{References}

1. Giove GC, Singla I, Mishra J, Nanda NC. Transesophageal echocardiographic finding of left atrial appendage lobe mimicking a mass lesion. Echocardiography 2011;28:684-5.

2. Levy-Ravetch M, Auh YH, Rubenstein WA, et al. CT of the pericardial recesses. Am J Roentgenol 1985;144:707-14.

3. Karakus G, Kodali V, Inamdar V, et al. Comparative assessment of left atrial appendage by transesophageal and combined two- and three-dimensional transthoracic echocardiography. Echocardiography 2008;25:918-24.

4. Okura K, Maeno K, Okura S, Takemori H, et al. Pericardial fat volume is an independent risk factor for the severity of coronary artery disease in patients with preserved ejection fraction. J Cardiol 2015;65:37.41

5. Albulushi A, Khan F, Al-Saidi K, Porter. T An unusual case of cardiac mass. Oman Med J 2018;33:176-7. 